MONOGRÁFICO: Materiales, transporte y producción. Pósters del Workshop Internacional de Arqueología de la Construcción V, (Universidad de Oxford, 11-12 Abril 2015 / MONOGRAPH: Materials, transport and production. Posters of the 5th International Workshop on the Archaeology of Roman Construction, (University of Oxford, April 11-12, 2015)

\title{
Refuerzo de fustes de columna en la arquitectura monumental de Italica (prov. Baetica). Aplicación de nuevas tecnologías para la reconstrucción de una práctica singular
}

\author{
Reinforced column shafts from the monumental architecture of Italica \\ (prov. Baetica). Implementation of new technologies for the reconstruction \\ of an exceptional practice
}

Oliva Rodríguez Gutiérreza ${ }^{a}$ Jesús Rodríguez Medina ${ }^{b}$, Francisco S. Pinto Puerto` y Roque Angulo Fornos ${ }^{c}$

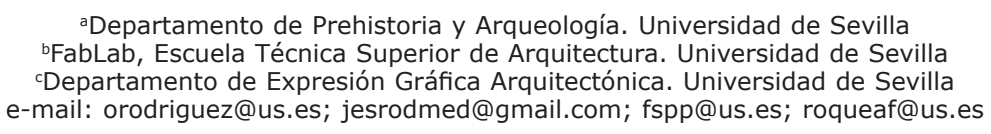

\section{RESUMEN}

Esta investigación tiene por objeto presentar los trabajos en curso en torno a un conjunto de fustes procedente de los órdenes arquitectónicos del conocido como Traianeum de Itálica (provincia Baetica, actual Santiponce, Sevilla), complejo de templo central y pórtico perimetral levantado en la ciudad a comienzos del siglo II d. C. En ellos se observan las huellas dejadas por toda una serie de recursos técnicos tales como grapas, espigas metálicas y cavidades para parches marmóreos, asociada con medidas preventivas de refuerzo de piedra eventualmente quebradiza. Para ello, dado su estado fragmentado y fragmentario, se ha recurrido a la aplicación de técnicas fotogramétricas que permitan la obtención de modelos tridimensionales de las piezas, con el fin de reconstruir el funcionamiento y finalidad mecánica de los citados recursos. En último término, se trata de profundizar en aspectos relacionados con una singular capacidad tecnológica selectiva y con la economía y organización de las obras.

Palabras clave: tecnología antigua; arquitectura romana; medidas preventivas; fotogrametría; restitución geométrica; modelización; programación visual; diseño paramétrico.

\section{ABSTRACT}

The pieces under study presented in this paper come from the already well known "Traianeum" of Italica (prov. Baetica, present Santiponce, Seville), a huge complex area, formed by a wide open square with a main central temple, built according to the extension of the city with a new quarter in Hadrian times. Most of them, fragments of column shafts, offer very peculiar technical features: canals for metallic dowels, cavities for marble tasselli and holes for also metallic staples or cramps. All these systems had the aim of reinforce a valuable material with potential structure problems. The ensemble is unique because of the preservation of a sufficient amount of fragments that allows their complete restitution with the help of photogrammetric techniques in order to obtain three-dimensional models of the pieces and to understand the ancient technologic solutions. Even more, the practices make us reflect about technical skills and economy and organization of constructions.

Keywords: ancient technology; Roman architecture; preventive measures; photogrammetry; geometric restitution; visual programming; parametric design.

Recibido: 09-06-2016. Aceptado: 16-09-2016.

Cómo citar este artículo / Citation

Rodríguez, O., Rodríguez, J., Pinto, F. S. y Angulo, R. 2016: "Refuerzo de fustes de columna en la arquitectura monumental de Italica (prov. Baetica). Aplicación de nuevas tecnologías para la reconstrucción de una práctica singular", Arqueología de la Arquitectura, 13: e048. doi: http://dx.doi. org/10.3989/arq.arqt.2016.166

\section{Copyright}

(C) 2016 CSIC. Este es un artículo de acceso abierto distribuido bajo los términos de una licencia de uso y distribución Creative Commons Attribution (CC-by) España 3.0 . 


\section{INTRODUCCIÓN}

Las piezas objeto de este estudio proceden del bien conocido "Traineum" de la ciudad romana de Italica (Santiponce, Sevilla, España, antigua provincia Baetica), una gran área monumental, constituida por una plaza abierta porticada con un templo central, construida con motivo de la ampliación de la ciudad hacia el norte, en tiempos del emperador Adriano (Fig. 1). El área, lamentablemente, se encuentra hoy mal conservada debido al intenso expolio sufrido por sus materiales de construcción, así como, incluso, al hecho de que aún la atraviese un antiguo viario público de servidumbre al cementerio del pueblo actual (Fig. 2). No obstante, los restos de las cimentaciones han podido ser bien identificados en el curso de excavaciones arqueológicas llevadas a cabo en la década de los ochenta del siglo pasado (León 1988), lo que ha propiciado la restitución de la totalidad de su planta. Del elevado número de columnas asociadas tanto al edificio de culto como al pórtico perimetral y al cuerpo monumental de acceso a éste último, sólo se ha recuperado un limitado número de fragmentos, abandonados en sus inmediaciones.

Al menos tres fustes monolíticos de columna en mármol portasanta y uno en cipollino verde, ambas variedades con origen en el Mediterráneo oriental, ofrecen rasgos técnicos peculiares (Fig. 3). En los diferentes fragmentos conservados se reconocen, con claridad, los restos de cavidades longitudinales cilíndricas para espigas metálicas internas, oquedades para grapas del mismo material y mortajas para parches (tasselli) marmóreos. Todos estos recursos tendrían el objetivo de reforzar, quizá ya en cantera $\mathrm{o}$, más probablemente, en un taller asociado a la obra, un material valioso pero cuyas características podrían suponer un peligro potencial de carácter estructural, con graves consecuencias para la estabilidad y perdurabilidad del conjunto.

Dicha práctica, documentada en otros edificios de la ciudad, como el teatro, así como en otros puntos del Imperio, incluida la propia Roma, ha sido analizada con anterioridad por nosotros (Rodríguez 2001, 2015) y parece, por tanto, bien caracterizada en líneas generales. La mayor parte de la bibliografía disponible, aunque

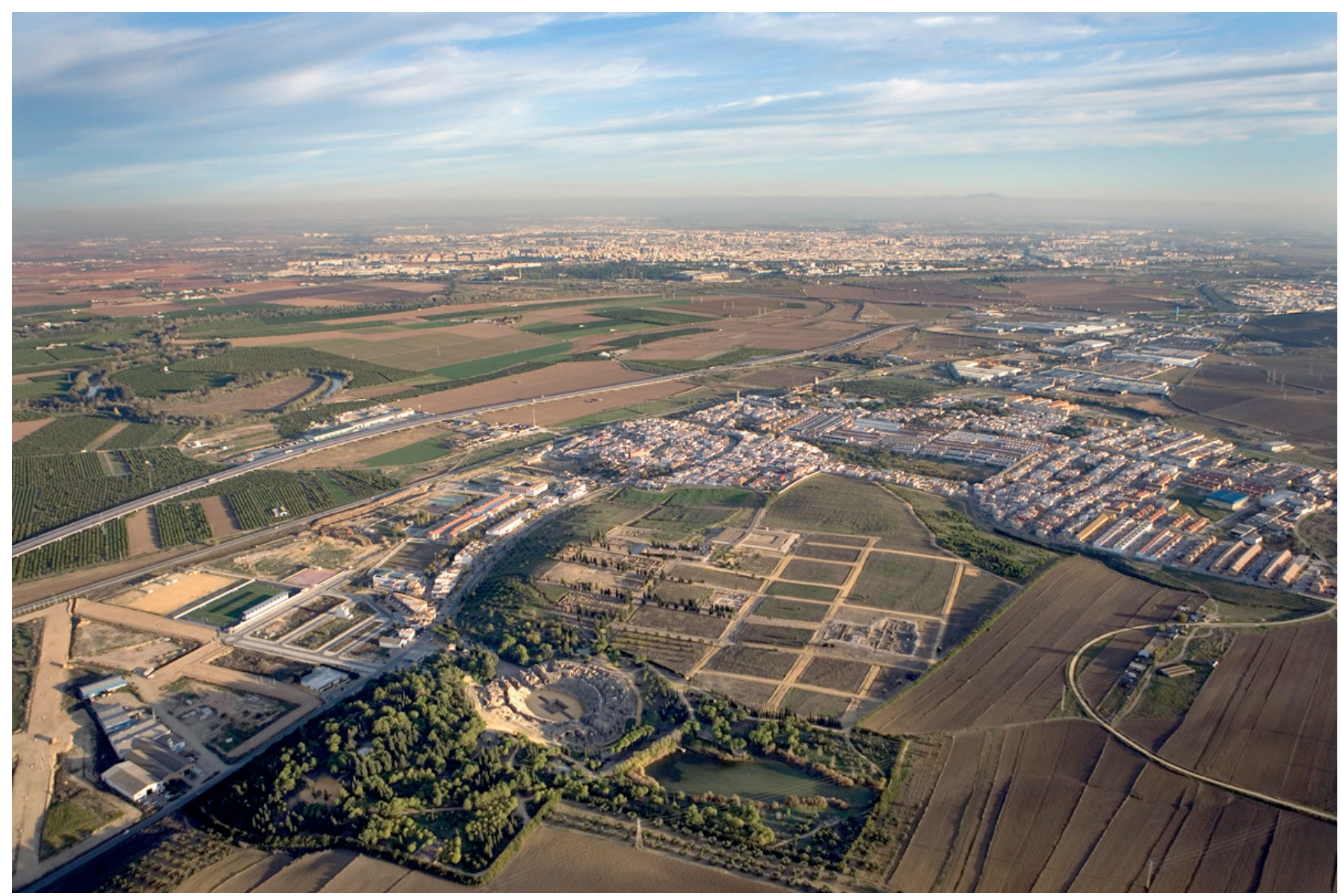

Fig. 1. Vista general actual de la ciudad romana de Itálica, desde el norte; en primer término, la ampliación de tiempos adrianeos (Fot. J. Hernández para Rutas del Teatro en Andalucía, Consejería de Cultura, Junta de Andalucía). 


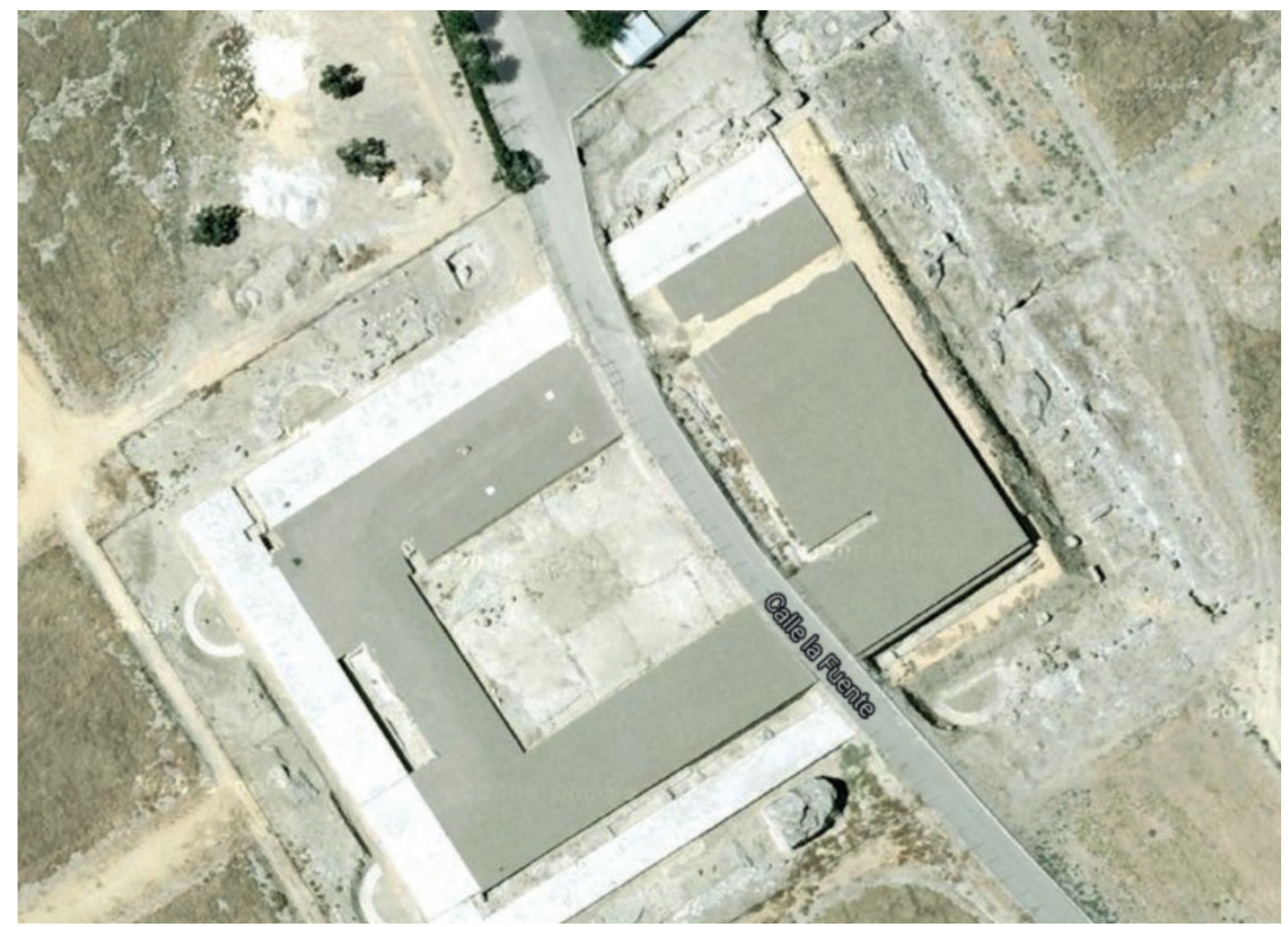

Fig. 2. Vista aérea del estado actual que presentan los restos del área monumental conocida como Traiaeneum. (Fot. Google Maps, CC).

minoritaria en conjunto, hace alusión a evidencias de ensamblaje entre elementos de la arqueología modular romana (Amici 2011, 2015: 66-67, 2016; Voigts 2012: $150-153$ y $160-163$, e. p.), cuando no a testimonios de restauraciones antiguas fruto o no, de dinámicas de reutilización (Ismaelli 2013), en ocasiones asociadas a medidas antisísmicas de las que ya se tenía conciencia en la antigüedad. Con frecuencia, los investigadores no han prestado a estos indicios la suficiente atención, en la medida en la que los han tenido por testimonios de reutilizaciones propias de la tardoantigüedad o la época medieval, cuando no, de actividades asociadas a expolio o restauraciones recientes.

En cualquier caso, las piezas del Traianeum, frente a los ejemplos estudiados de otros contextos, son únicas, en la medida en la que se ha preservado un número suficiente de fragmentos de los fustes originales para permitir una propuesta de restitución bastante completa de cada uno de los ejemplares. Ello, no obstante, dada su complejidad, volumen, peso y estado fragmentario, está siendo posible valiéndose de nuevas posibilidades de aplicación tecnológica sólo hoy a nuestra disposición. De esta forma, las necesidades de estudio e interpretación de esta singular y aparentemente atípica práctica antigua, son las que han llevado a buscar auxilio en nuevas herramientas susceptibles de aplicación a estas cuestiones de índole histórico y patrimonial.

De esta forma, el presente estudio aborda, de forma concreta, la incorporación a la investigación sobre estas singulares soluciones de la arquitectura romana de técnicas de programación visual y diseño paramétrico, aplicadas al análisis comparativo de modelos tridimensionales obtenidos a partir de capturas fotogramétricas (con imágenes capturadas por UAVs o dron) y modelos de geometría teórica. Ello facilita la reconstrucción completa de cada uno de los fragmentos con la precisión suficiente que posibilite recoger, con detalle, todos los rasgos técnicos que contienen. El grado de desarrollo de estos instrumentos, frente a versiones más antiguas, permite hoy, incluso, dotar a las nubes de puntos del aspecto real de los volúmenes originales. Esto es especialmente valioso en este caso, dado que, como se verá más adelante (Fig. 4), los rasgos técnicos parecen tener directa relación con las características estructurales del mármol, reflejadas en sus vetas, matriz y grano. De esta forma, será posible proponer la hipótesis de funcionamiento de 


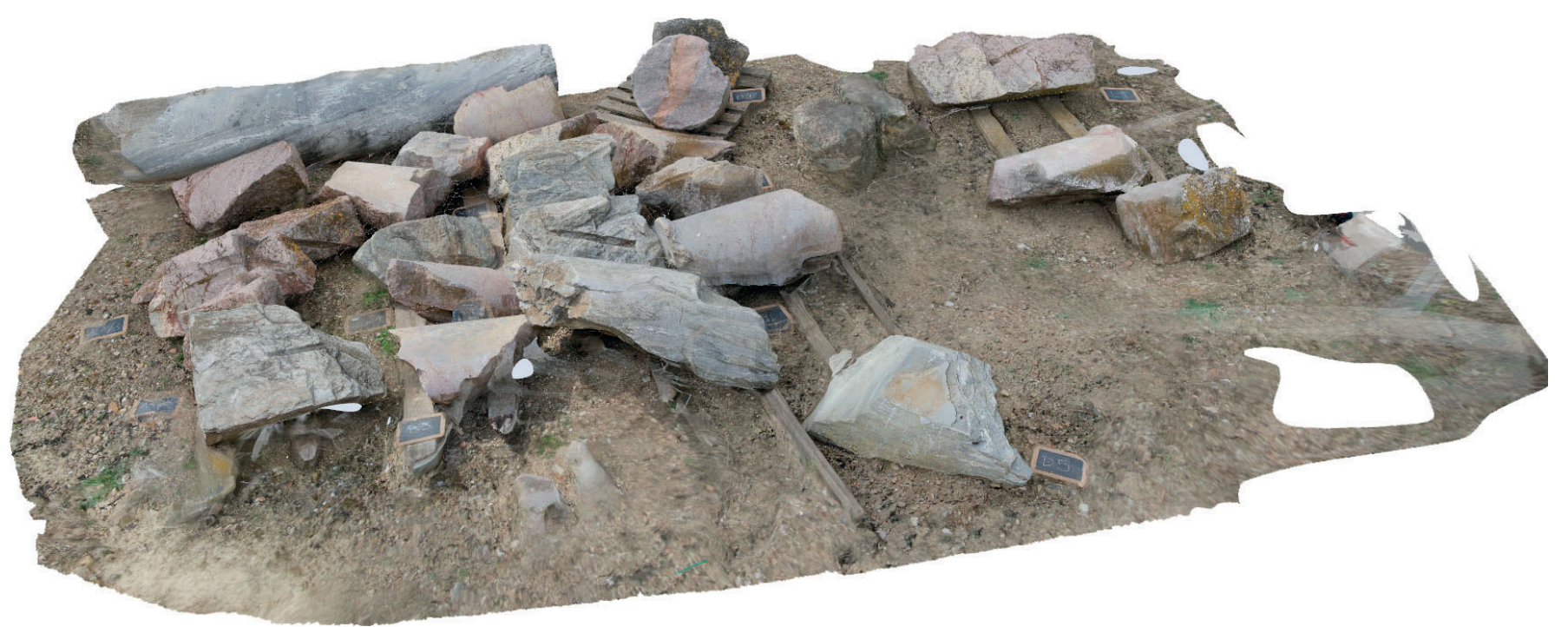

Fig. 3. Imagen de parte de las piezas objeto estudio, generada a partir de la fotogrametría de detalle de cada uno de los fragmentos.

las espigas, grapas y parches, planteando su finalidad y la eficacia de su empleo. Dada no solamente la cantidad de metal empleada sino, más aún, la complejidad técnica requerida, parece indudable que se trató de un procedimiento llevado a cabo por cualificados operarios, óptimos conocedores del material y su comportamiento. Más aún, dicha práctica hace reflexionar sobre los eventuales costes de extracción y transformación de piezas de gran porte en cantera para arquitectura monumental, en la medida en la que se prefirió su refuerzo a su descarte e, incluso, se hicieron llegar hasta una relevante ciudad del Occidente romano teniendo en cuenta los elevados gastos de transporte para su empleo en un proyecto de arquitectura pública monumental.

\section{CONTEXTO GEOGRÁFICO E HISTÓRICO}

La ciudad romana de Itálica (provincia Baetica, actual Santiponce, Sevilla), en el fértil valle el Guadalquivir, a pesar de haber sido ya fundada a fines del siglo III a. C. y contar con antecedentes turdetanos, se hizo especialmente célebre por ser la patria del primer emperador provincial, Trajano. La tradición literaria insistía, además, en los vínculos que con la ciudad habría siempre mantenido Adriano, su sucesor. Con él, tradicionalmente, incluso en forma de una implicación directa no constatada con seguridad, se ha asociado una imponente obra de ampliación de la ciudad que la extendió hacia el norte en varias hectáreas (Fig. 1). Durante más de

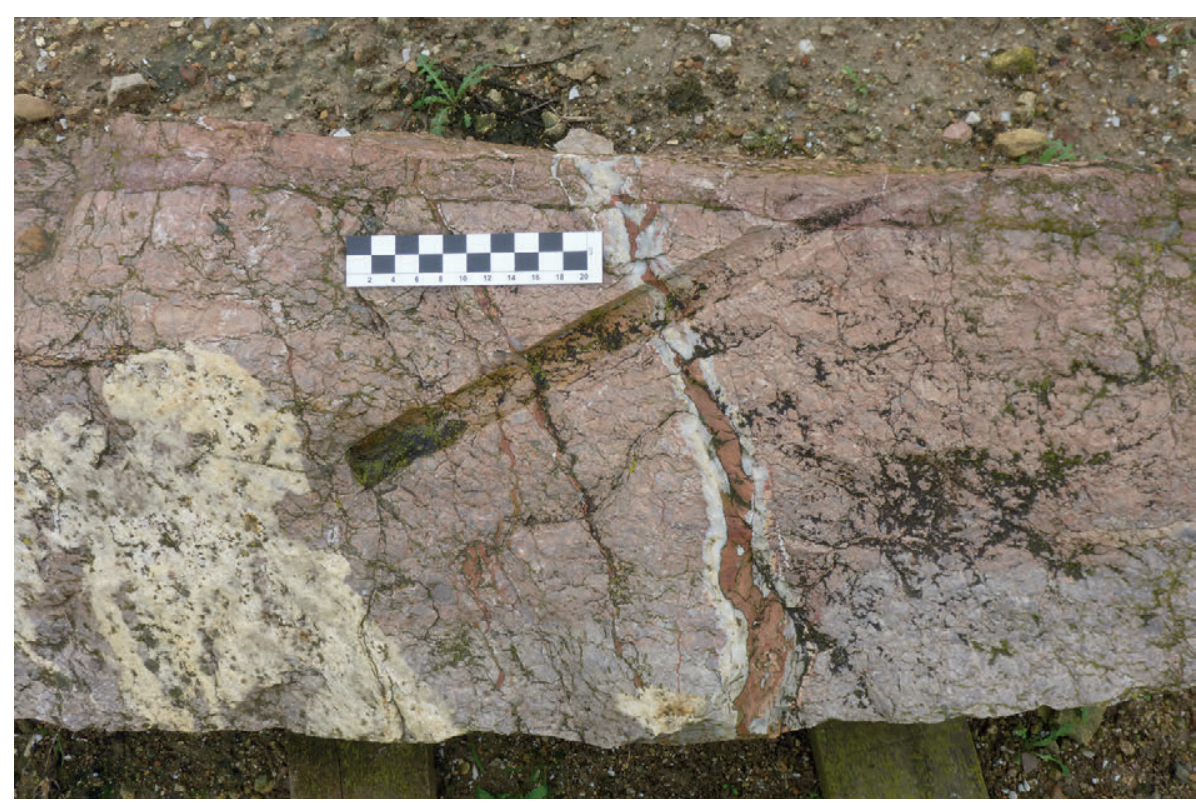

Fig. 4. Fragmento de fuste con cavidad para espiga metálica longitudinal, insertada de forma perpendicular a las vetas del mármol. 
dos siglos los trabajos arqueológicos se han sucedido en su solar, hoy correspondiente, a grandes rasgos, con la entidad patrimonial del Conjunto Arqueológico de Itálica. En el área, buena parte amurallada, se reconocen no solamente diferentes unidades domésticas de grandes dimensiones y ricos acabados, sino también notables edificios públicos (Caballos 2010). Entre ellos destacan unas termas con palestra, el anfiteatro y un gran espacio porticado con templo central, conocido en la bibliografía reciente como Traianeum, por considerarse un recinto de culto imperial levantado por Adriano en honor de su antecesor (Fig. 2), hipótesis que, por el momento, se mantiene, a pesar de no existir pruebas de ello todo lo sólidas que sería deseable.

\section{EL EDIFICIO Y SUS MATERIALES}

Es, precisamente, de este último complejo del que proceden las piezas objeto de este estudio. Los trabajos de análisis y restitución del edificio (Montero en León 1988: 91-101) han permitido reconstruir el número, módulo y características de los principales órdenes arquitectónicos: la columnatio perimetral del pórtico presentaría 20 columnas en sus lados cortos y 28 en los largos, con una fachada monumental hexástila en el eje axial. En el primer caso los fustes, combinados con basas y capiteles en mármol blanco de Luni, estarían realizados, al menos, en mármol cipollino (Eubea), en sus variantes gris y verde ( $\varnothing 63 / 65 \mathrm{~cm}$ ); la fachada monumental de acceso, a su vez, se resolvería con grandes fustes rosados en mármol portasanta (Chíos), de en torno a $72 \mathrm{~cm}$ de diámetro.

Sin embargo, de ese abundante número de elementos arquitectónicos originales han sido muy pocos los recuperados, ya sea a partir de las excavaciones realizadas en la década de los ochenta del siglo pasado o, más aún, abandonados en el área, objeto de rebuscas en diferentes momentos. En cualquier caso, el expolio intenso ha sido generalizado en el edificio, que hoy tan sólo puede ser reconocido al nivel de sus cimientos.

En cualquier caso, de las escasas piezas documentadas, hoy localizadas en el entorno directo del Traianeum y sin contextos arqueológicos claros de hallazgo ${ }^{1}$,

\footnotetext{
1 Frente a ello, en el teatro y su parte superior sí han sido hallados ejemplares en contextos estratigráficos precisos, que remiten a fases tardías (s. V d. C.) de expolio y reutilización de materiales altoimperiales (Rodríguez 2015: 366-368). El hecho de que el registro provenga de diferentes edificios, localizaciones y contextos parece poder corroborar, sin duda, su carácter de práctica antigua.
}

un elevado número de fragmentos presenta diferentes recursos técnicos (Fig. 3). Han sido identificados, al menos, en fustes de mármol portasanta ${ }^{2}$ y cipollino verde. Uno de los aspectos relevantes que podrá ser confirmado a través del ensayo en curso es, precisamente, el número mínimo de individuos, dado que se logrará ubicar los diferentes fragmentos en sus posiciones concretas en la geometría completa de los fustes.

Los diferentes rasgos técnicos, a pesar de las ligeras variantes, pueden simplificarse en tres categorías principales que, a su vez, pueden aparecer combinadas entre sí:

- Largas espigas metálicas cilíndricas (Figs. 4 y 5). De ellas resta el canal del barreno o sierra cilíndrica ${ }^{3}$ (Voigts 2012: 151), que no trépano, para su inclusión con, ocasionalmente, restos de metal (hierro) ${ }^{4}$. Las barras, a diferencia de otros ejemplos estudiados (Amici 2015: 66-67), en ningún caso parecen ubicarse en el eje axial vertical de los fustes, sino que son oblicuas y excéntricas. En muchos casos se documenta el final de la espigas alojado en el interior de las piezas; por tanto, no las atravesaban de lado a lado. A su vez, fueron incluidas de forma perpendicular a las vetas del material y no a favor de ellas (Fig. 4), lo que parece descartar su identificación como recursos para la reutilización de material constructivo en bruto en época reciente.

- Grapas o tirantes metálicos en forma de "pi" de longitud diferencial, alojadas en mortajas realizadas en la piedra a tal efecto.

- Cavidades de diferentes formas pero con contornos lobulados $^{5} \mathrm{y}$ fondo generalmente desbastado a base

\footnotetext{
Un significativo ejemplo de fuste en el mismo material, reforzado al interior con espigas metálicas verticales procede del orden del pórtico de la palestra oriental de las termas de Trajano en Roma (Amici 2015: 67).

3 Evidencias formalmente muy semejantes han sido identificadas por C. Voigts (e. p.) en diferentes materiales arquitectónicos de la Domus Augustana (piezas de entablamento) y la Domus Flavia del Palatino (capitel corintio), aunque de nuevo, en esta ocasión, para favorecer una adecuada tracción entre elementos independientes. No deja de ser significativo que la datación de dichos recursos se sitúe a comienzos de la segunda centuria lo que parece confirmar una pericia técnica consolidada.

4 En numerosas ocasiones se ha documentado la cobertura de las grapas y pernos de hierro con una capa de plomo, recurso que las protegía de la oxidación, evitando así que los propios tirantes, al degradarse, generaran dañinas presiones internas.

Que, desde el punto de vista técnico y formal, podría corresponder a los emblemata del tipo C.2 de Ismaelli (2013: 278), D, en caso de reforzarse las uniones con elementos metálicos. No obstante, este autor, para su coherente clasificación parte, en prácticamente todos los casos, de su carácter de reparaciones de daños efectivos, por lo que se distanciaría, en cierto sentido, de la interpretación de los casos italicenses, preventivos. En cualquier caso,
} 


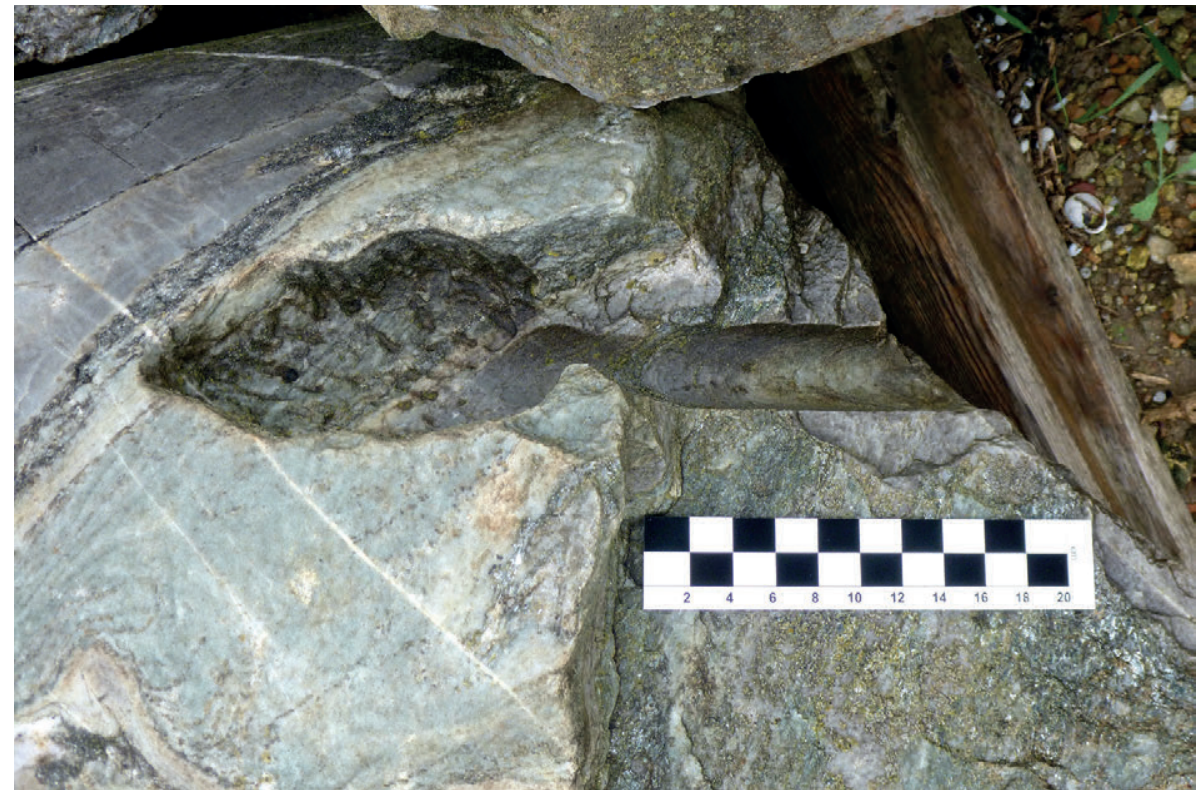

Fig. 5. Cavidad para parche marmóreo en el remate de una espiga metálica. de puntos de pico a fin de generar superficie de anathyrosis; finos pernos metálicos internos podían, asimismo, reforzar las uniones ${ }^{6}$. Aunque pueden aparecer aisladas, con frecuencia su función será la de ocultar, en la superficie vista de los fustes, la inclusión de espigas y grapas (Fig. 6).

Estas prácticas han sido documentadas y estudiadas en otros edificios italicenses como el teatro (Rodríguez 2001) y su área superior (Rodríguez 2015: 366-368), lo que parece constatar su relativa difusión en la ciudad. Lamentablemente, a pesar de su interés, se trata de recursos que, de forma general, apenas han llamado la atención de los investigadores de la arquitectura romana en otros puntos del Imperio. Ello se debe a que, de forma un tanto simplista, han tendido a leerse como fruto de dinámicas de reempleo y reutilización de espacios y materiales de época tardoantigua en adelante y no, por tanto, coetáneos al uso original de las piezas. En el mejor de los casos, se han interpretado como reparaciones y/o restauraciones de piezas dañadas ya sea de antiguo

en dicha variante incluye los conocidos ejemplos ostienses que citamos más adelante, identificándola como una solución itálica, incluso metropolitana, con cierto predicamento en los territorios occidentales del Imperio (Ismaelli 2013: 286) y sin apenas repercusión en Oriente, área objeto de su detallado estudio.

6 Junto a recursos de anathyrosis y pernos se ha documentado (Ismaelli 2013: 278), además, el uso de sustancias adherentes para reforzar las uniones de piezas: cera de abeja, argamasas, arcillas, caseínas. De ello, lamentablemente, no se han encontrado evidencias en los ejemplos italicenses, pero cabe suponerlo en el caso de parches sin recursos de fijación lo suficientemente eficaces. o, incluso, en época reciente ${ }^{7}$. Ello quizá se haya debido a la perfección técnica en la ejecución de estos recursos, especialmente de las cavidades cilíndricas. Para ello se empleó, como ya se ha indicado y constan ejemplos metropolitanos (Voigts 2012), una potente barrena circular que cortaba el mármol por presión mecánica, dejando incluso, en el fondo de algunas de estas perforaciones la huella del anillo cortante.

Casi como única excepción a las anteriores interpretaciones cabe citar una serie de ejemplares procedente de Ostia, con rasgos muy similares (Baccini Leotardi 1979; 1989; Pensabene 1995), casos que parecen poder ampliarse en número con el creciente interés de los investigadores por estos aspectos técnicos. Así, T. Ismaelli ha identificado evidencias en ciudades de Asia Menor como Klaros (2013: 276), Éfeso y Nysa (2013: 277), sobre bloques reforzados en la propia obra, una vez que los trabajadores tomaron conciencia del peligro de roturas potenciales. También en cantera se documentan fustes con intentos de refuerzo por medio de grapas/tirantes metálicos, si bien, a pesar de ello, finalizaron por ser allí abandonados (Wurch-Kozelj 1988: 55; Ismaelli 2013: 277).

Del análisis pormenorizado de las piezas se ha llegado a la conclusión de que estos rasgos no solamente son coetáneos a su momento de utilización sino, más aún, que se realizaron a fin de reforzarlas, con fines preventivos, con anterioridad a la colocación en el

\footnotetext{
Como hace también notar C. M. Amici para el caso de Villa Adriana (Amici 2011: 223, fig. 3).
} 


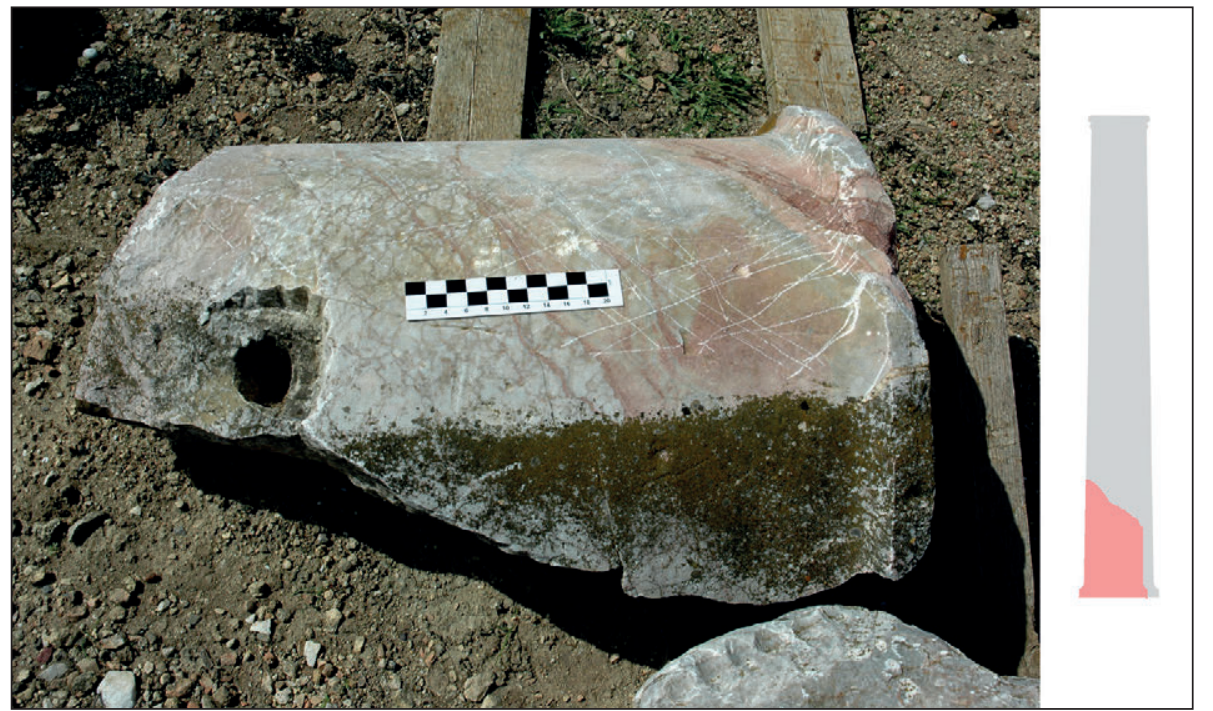

Fig. 6. Cavidad para parche marmóreo con encaje interno de sección cuadrada. edificio. Éste se trata, sin duda, de un fenómeno bien conocido en la arquitectura antigua para garantizar la cohesión de las estructuras construidas articuladas (Amici 2011: 227), aunque no deja de ser excepcional en casos como el que nos ocupa, al interior de elementos monolíticos sin función de ensamblaje entre piezas independientes. No obstante, parece tratarse de soluciones de elevada sofisticación técnica que no alteraban la percepción visual de la integridad del material.

Visto todo lo anterior y dado que nada llevaba a pensar, a partir de la actual observación de los fragmentos conservados, en roturas efectivas, se hacía preciso tratar de entender la trascendencia estructural de estos recursos en el contexto de las piezas completas, tanto en lo referido a la naturaleza del material pétreo como a la función estática desempeñada por los fustes.

\section{LA METODOLOGÍA DE TRABAJO}

Partíamos de la experiencia previa de la restitución y modelado de los numerosos fragmentos de columnas conservados de la scaneae frons del teatro de Itálica con objeto de diseñar su anastylosis (Pinto, Guerrero y Angulo 2011). En esa ocasión se planteó la necesidad de obtener un conocimiento de las características métricas de los fragmentos con objeto de verificar, una vez modelados digitalmente, las hipótesis existentes sobre sus tamaños y composición sobre el conjunto murario del teatro. La exactitud en la modelización digital garantizó la obtención de medidas ciertas de diámetros, curvatura de éntasis y encaje de las roturas, sin necesidad de mover una gran cantidad de masas de piedra de su actual localización, con unos recursos técnicos y económicos discretos.

Una vez establecida como punto de partida esta primera experiencia, la metodología seguida supone una evolución del método empleado en aquel trabajo mediante la incorporación de nuevas técnicas de análisis digital basadas en herramientas de programación visual y diseño paramétrico. Éstas introducen una importante innovación respecto a los procesos que en aquel momento se emplearon para el reconocimiento geométrico y formal, a partir de la restitución tridimensional de los diferentes fragmentos conservados, correspondientes, en este caso, a fustes de los citados materiales y módulos. Así, el avance propuesto para el trabajo que ahora presentamos es el de incorporar, por un lado, la parametrización de la geometría de los modelos digitales de los fragmentos, es decir, la incorporación de un serie de variables numéricas susceptibles de ser relacionadas entre sí y con otros valores externos cuya manipulación permite su control geométrico; y, por otro, la introducción de la programación visual como herramienta capaz de controlar dichos parámetros y aprovechar las ventajas de la computación para la realización, en tiempos insignificantes, de múltiples cálculos y procesos ${ }^{8}$, destinados a facilitar el análisis geométrico y material de los fustes. Por otro lado, se ha mejorado el procedimiento

\footnotetext{
En la citada experiencia del teatro, la obtención de la geometría teórica asociada a cada pieza consistía en la realización de múltiples tanteos realizados manualmente mediante herramientas gráficas basadas en CAD.
} 
de obtención de cualidades liminares, fundamentales para relacionar las piezas y valorar la utilidad de los refuerzos aplicados en épocas antiguas.

Las piezas han sido descritas e inventariadas, así como fotografiadas desde todos los ángulos posibles a través de imágenes digitales capturadas de forma convencional y con tecnología $U A V$. A partir de dichas imágenes, a través de la aplicación de la fotogrametría digital, se han generado modelos $3 \mathrm{D}$ texturizados de cada uno de los fragmentos (Fig. 7).

Mediante la aplicación de las nuevas técnicas incorporadas se han realizado los análisis métricos precisos de las piezas conservadas, así como su cotejo con los principios de proporciones convencionales
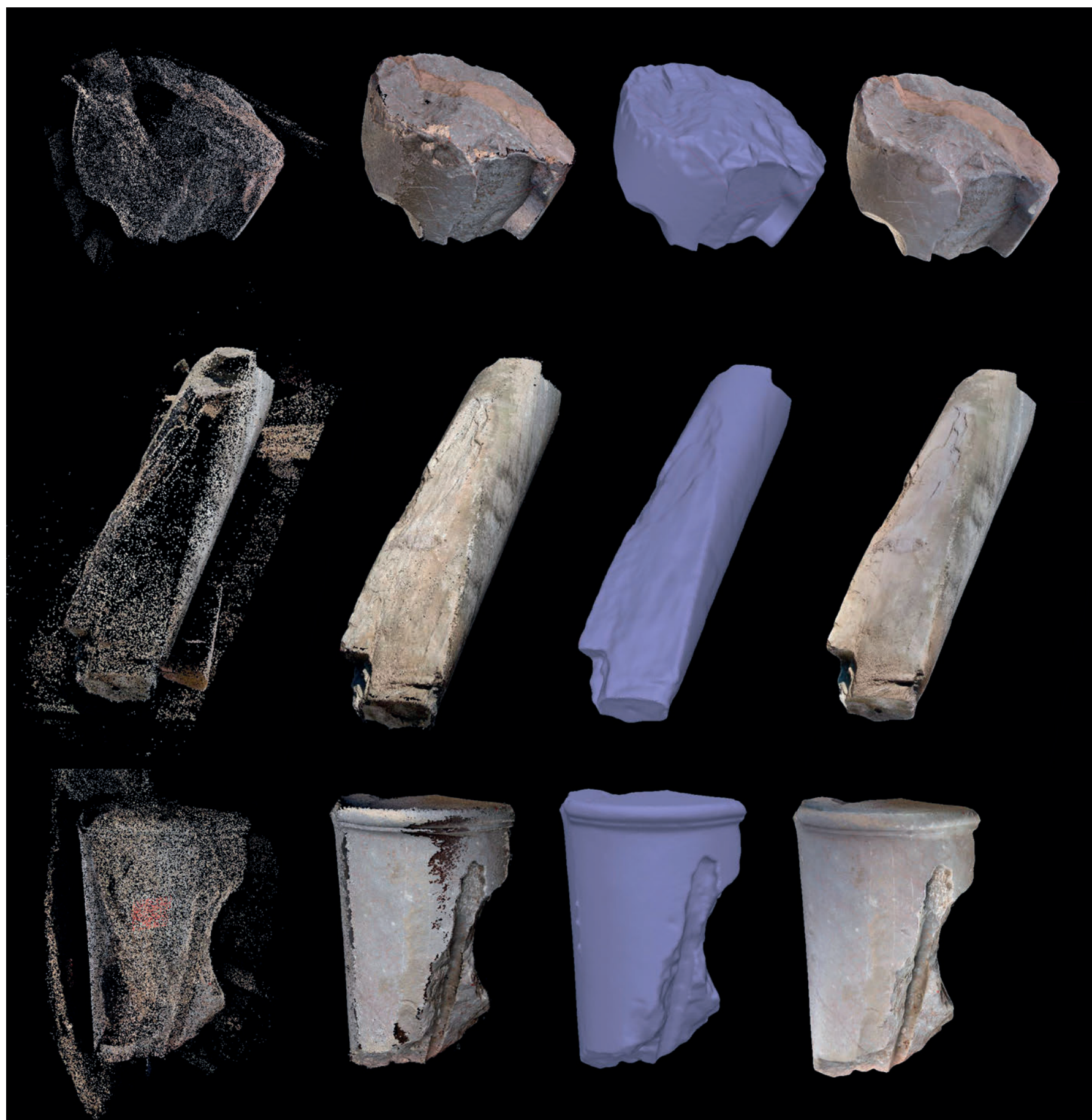

Fig. 7. Ejemplo de generación del modelo tridimensional de algunas de las piezas a partir de la nube de puntos inicial. 
para los órdenes en arquitectura romana, obteniendo como resultado la estructura geométrica de referencia de los fustes. Una vez conocida ésta se ha procedido, a través de los cálculos geométricos pertinentes, a la integración de los fragmentos en ella, a fin de contextualizar en los elementos arquitectónicos completos los recursos técnicos identificados y poder llevar así a cabo su interpretación (Fig. 8).
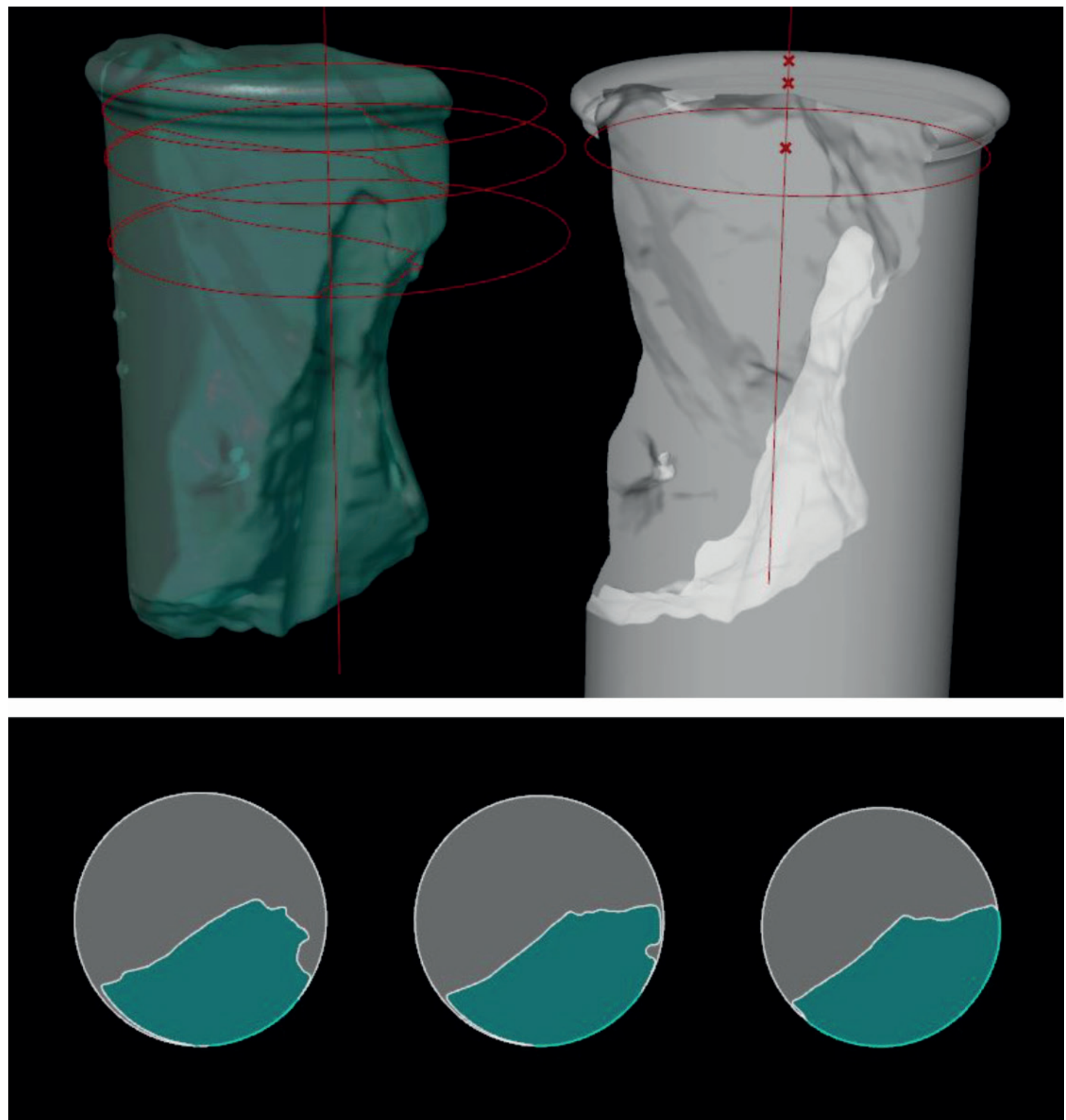

Fig. 8. Ensayo de contextualización de fragmento con recursos técnicos en la geometría ideal de un fuste monolítico del módulo correspondiente. 


\section{Captura métrica mediante fotogrametría digital de las piezas}

La irregularidad en las roturas que presentan muchas de las piezas ha hecho necesario recurrir a las últimas técnicas desarrolladas en el ámbito del levantamiento arquitectónico. A partir de éstas, hemos podido producir modelos tridimensionales de geometría precisa y portadores de atributos visuales suficientes (color, textura, marcas, etc.) como para permitir un adecuado análisis de la configuración geométrica y material de las mismas.

El proceso se ha basado en los siguientes hitos (Fig. 7):

- Captura de nubes de puntos mediante fotogrametría digital. A partir de tomas fotográficas de conjunto ${ }^{9}$ y detalle, y de algunos datos métricos obtenidos de forma tradicional (no era necesario recurrir a la topografía para georreferenciar los modelos), se generaron nubes densas de puntos mediante el software PhotoScan (C) Agisoft).

- Tratamiento de nubes en el software Meshlab para su limpieza y conversión en mallas de triángulos optimizadas.

- Importación de mallas en Photoscan para la generación de texturas y la exportación de los modelos tridimensionales definitivos.

\section{Ichnographia de las piezas arquitectónicas}

Con objeto de avanzar en el conocimiento de las características geométricas y formales de las marcas y roturas de las piezas se procedió al tratamiento de los modelos generados en la captura. Para ello se trabajó en un software de modelado digital en 3D, en concreto Rhinoceros (C) Robert McNeel \& Associates), construyendo sobre los modelos lo que viene a denominarse su ichnographia (Jiménez 1994: 29-71): generatrices, directrices, ejes de simetría, ejes de taladros, posición de cavidades, etc. (Pinto, Guerrero y Angulo 2011). Estos trazados geométricos realizados sobre los modelos 3D solventaban la dificultad de su obtención directa sobre las piezas, pero mantenían algunas de las problemáticas comunes en el estudio geométrico tradicional. En ambos casos no era posible la sistematización ágil y eficaz de un proceso que, como

\footnotetext{
9 El volumen de las piezas y, especialmente, su peso ha hecho que el auxilio de tecnología UAV para fotografías de conjunto haya sido de gran utilidad para completar la fotogrametría obtenida a partir de capturas de detalle.
}

en el caso que nos ocupa, basa gran parte de su utilidad en obtener información de una gran cantidad de piezas. El análisis de las relaciones entre los datos parciales de cada pieza de fuste debía hacerse mediante la elaboración de tablas comparativas que fueran recogiendo numerosos datos y relacionarlos numéricamente entre sí y con un modelo teórico del elemento estudiado. El método de trabajo que ahora se propone, que busca potenciar el análisis sistemático y complejo de las piezas, lleva a la utilización de herramientas de nueva generación basadas en la programación visual, es decir, a fin de poder establecer numerosas y complejas relaciones geométricas y formales directamente sobre el modelo. El recurso aplicado para ello es el plugin Grasshopper (Fig. 9) para el software Rhinoceros. Este recurso proporciona las herramientas necesarias para programar un script ${ }^{10}$ que, enfocado al problema tratado, permite profundizar, no sólo en el conocimiento de la pieza, sino también en una metodología de estudio basada en la manipulación directa de modelos con una alta precisión métrica. Lo novedoso de su aplicación es la sustitución de las limitadas manipulaciones posibles mediante un uso manual del CAD, por el diseño de un software personalizado capaz de realizar cientos de esas acciones en tiempos muy reducidos y de forma mucho más precisa. Así, esta herramienta, permite compensar las inexactitudes existes a menudo por lo orgánico de la pieza física frente al modelo teórico con el que se compara. De esta forma, la obtención y el análisis de una gran cantidad de información que nos proporcione medidas exactas, permite trabajar con el error como un parámetro más de análisis. Por ejemplo, a partir de tomas de datos con muchas muestras y las medidas de varios diámetros, considerando un margen de error determinado, podemos programar el software para que las compare y nos diga si las diferentes medidas tomadas varían según el éntasis de los tercios superiores del fuste, así como localizar los mayores pertenecientes al tercio recto inferior del fuste. Por otro lado, el método permite obtener medidas precisas de las piezas al facilitar el ajuste repetitivo de proporciones hasta alcanzar valores de error asimilables entre la pieza real y el modelo teórico. Este procedimiento de trabajo sobre las trazas geométricas nos ha permitido aproximarnos, por un lado, a las relaciones existentes entre las dimensiones de los fragmentos y sus posibles piezas complementarias y, por otro, al potencial origen de las manipulaciones antiguas detectadas.

\footnotetext{
10 Archivo de órdenes, guión o pequeño programa basado en un lenguaje de programación concreto, capaz de realizar diversas tareas como generar, editar y analizar grandes listados de datos, e interactuar con otros software, en este caso, de diseño asistido por ordenador.
} 


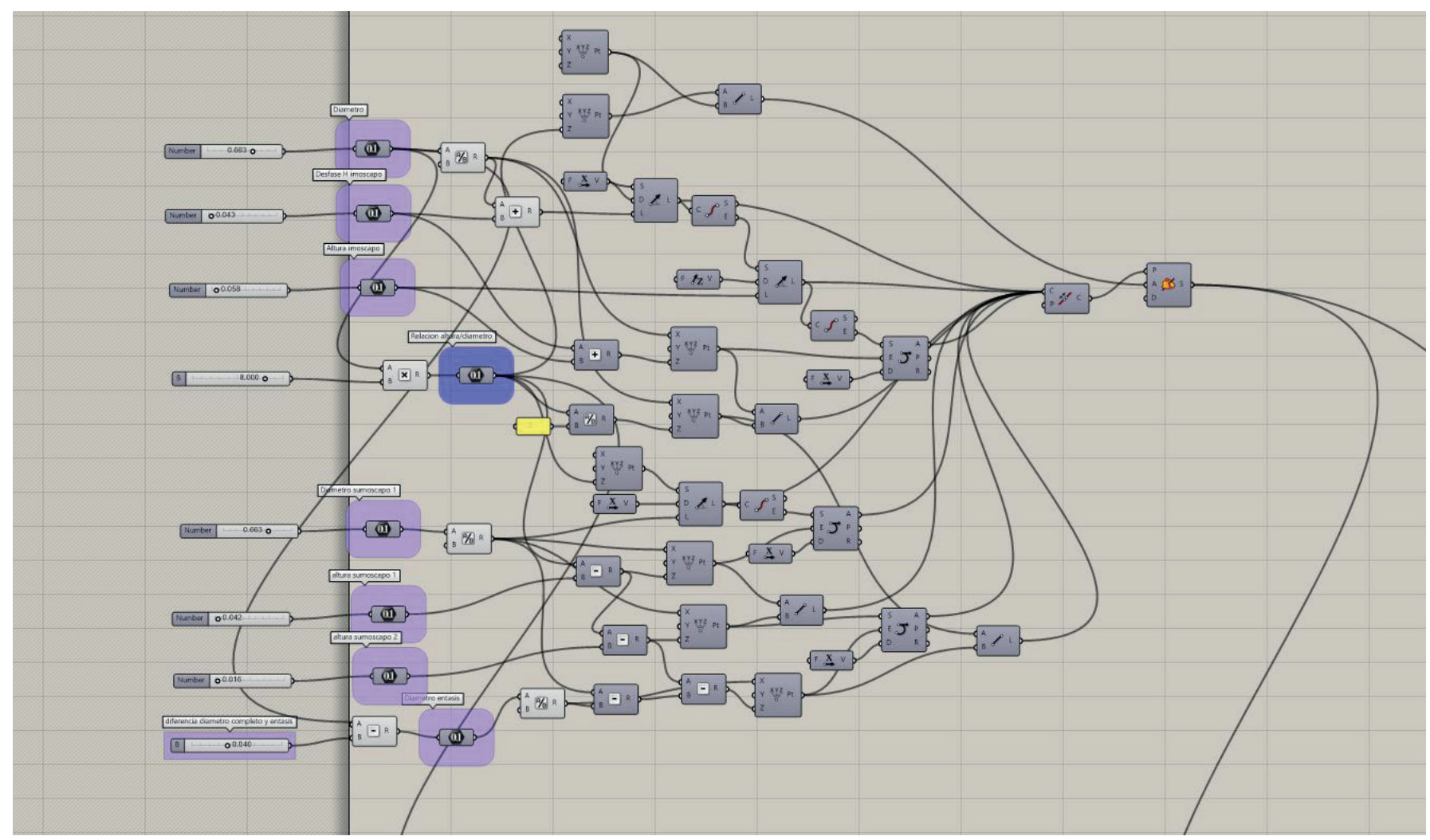

Fig. 9. Definición del script diseñado para el análisis de la ichnographia de las piezas.

\section{Análisis arquitectónico}

El proceso de análisis arquitectónico de cada pieza continúa con la comparación de cada fragmento con un modelo de fuste teórico, basado en los módulos y tipos definidos por la tratadística vitruviana (Jiménez 1989: 312). Este modelo teórico sirve así de soporte o base en el que ir verificando la posición de cada fragmento en función de las peculiaridades de sus atributos de figura, tamaño y orientación, es decir, por la presencia de imoscapo, sumoscapo, directrices rectas o curvadas de éntasis, que es posible medir con exactitud gracias al levantamiento realizado con los procedimientos antes expuestos. Una vez ajustados a este modelo teórico se verifican los atributos de color, textura y figura de las superficies que se han modelizado por medio de los recursos tecnológicos anteriores, es decir, coloración, veteados. Posteriormente se verifica el ajuste de las roturas y se procede a su encaje, comprobando así la pertenencia a una misma unidad o individuo, y la comprensión del funcionamiento conjunto con el objetivo de evaluar el alcance y trascendencia de los recursos técnicos, principal cometido de la investigación.
Como punto de partida se han tomado las medidas propuestas para el pórtico del Traianeum (Montero en León 1988: 58) de 5,30 m para la longitud del fuste, para un diámetro de $0,66 \mathrm{~m}$ medido en el tercio inferior (el más cercano al imoscapo), que responde a la propuesta de Vitruvio de entre 7,9 y 8 veces el diámetro. Estas proporciones serán a su vez modificadas para reajustarse a la realidad de los fragmentos conservados, hasta obtener un resultado óptimo. Estos mismos resultados se han podido verificar, en principio tan sólo de forma experimental, a través de impresiones 3D de las piezas merced a los recursos disponibles en el FabLab de la ETSA de la Universidad de Sevilla, pudiendo así obtener una maqueta a escala (Fig. 10).

\section{INTERPRETACIÓN}

En el conjunto templo-pórtico la combinación de diferentes marmora coloreados parece haber sido un recurso estético y simbólico de gran impacto. De este modo, las variedades foráneas elegidas para algunas de las series de fustes eran de gran singularidad cromática así como muy valoradas en el mundo romano, aunque 

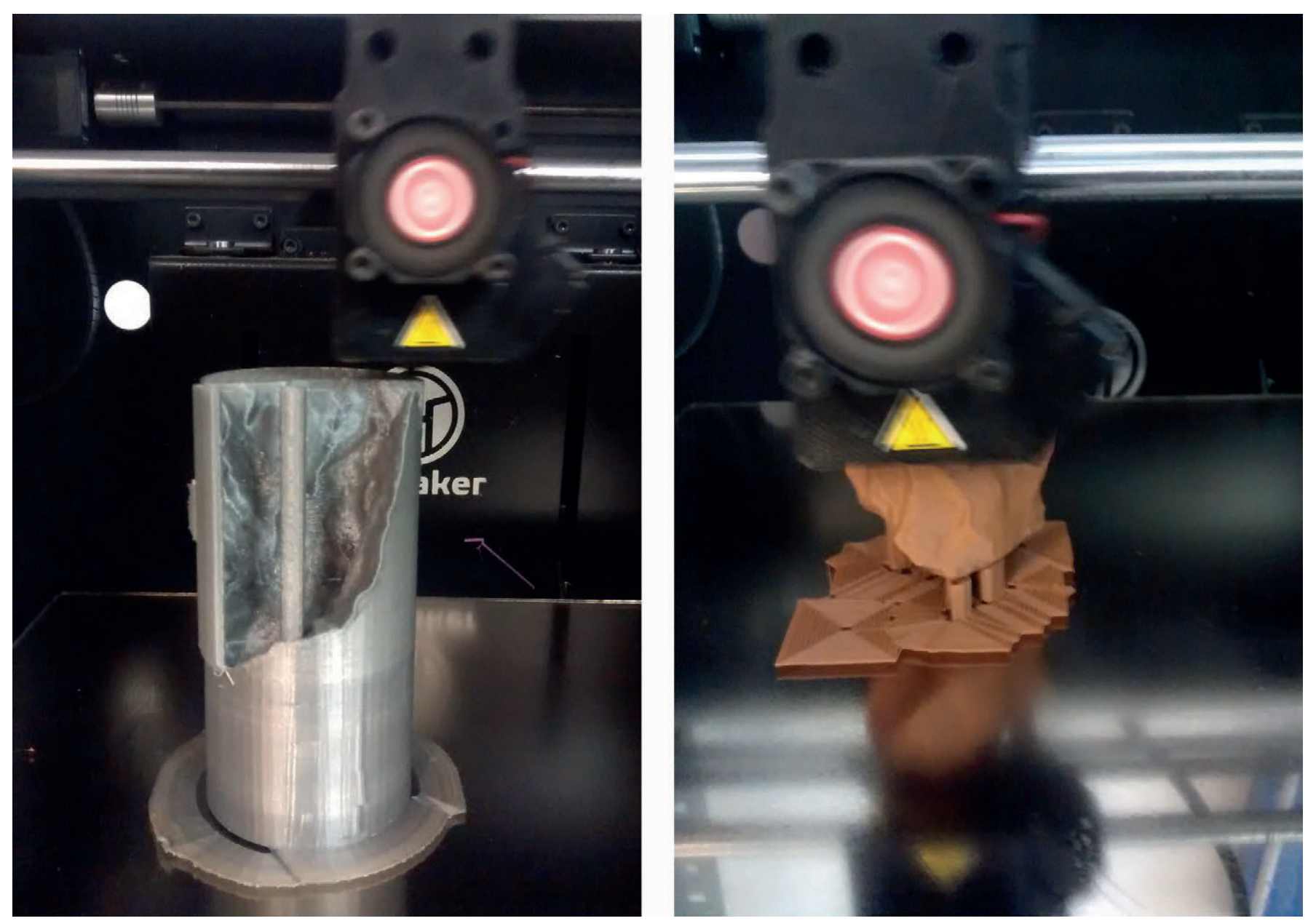

Fig. 10. Producción de maqueta a escala de la propuesta de restitución por medio de impresora 3D (FabLab ETSA).

su naturaleza estructural, con componentes de diferente dureza y compactación, podrían responder de forma diferencial a las presiones y empujes, una vez colocados en el edificio. Todo parece indicar que se habrían llevado a cabo complejas y costosas acciones de refuerzo de los elementos, a modo de "cosidos" internos, por medio de espigas, grapas y cuidados parches marmóreos lobulados para disimular las anteriores. No es posible precisar aún si estas acciones se llevaron a cabo ya en cantera, con anterioridad al transporte, o ya a pie de obra o en un taller asociado a ella. En ese sentido, el hecho de tratarse de recursos semejantes en materiales diferentes no resulta un dato dirimente, en la medida en la que las técnicas de cantería y ejecución de elementos se encontraban enormemente estandarizadas tanto en el espacio como en el tiempo. De hecho estas prácticas parecen poder enmarcarse en un momento de especial demanda de piedras ornamentales, en paralelo con un mayor perfeccionamiento de la pericia técnica tanto en cantera como en talleres de destino. No obstante, los resultados obtenidos de la restitución de las piezas en el curso de esta investigación lleva a pensar que los refuerzos se ejecutaran teniendo muy en cuenta las funciones estructurales y los valores tectónicos de los elementos, y esto sólo cabe esperarlo dentro de la propia dinámica de obra. En esa línea, diferentes estudios recientes (Amici 2011, 2015) insisten en el óptimo conocimiento que de la aplicación de elementos metálicos ${ }^{11}$ se llegó a tener en la arquitectura romana imperial, especialmente a partir de época de Augusto, para garantizar la cohesión de las construcciones modulares en piedra o también en opus caementicium. Estos recursos no habría necesariamente que contemplarlos exclusivamente como soluciones provocadas por imprevistos de obra, sino que en buena medida formarían ya parte de la planificación de la misma fruto de un largo recorrido en investigación aplicada

\footnotetext{
11 Entre sus principales propiedades (Amici 2011: 221) se encuentran la capacidad para resistir los empujes, aligerar las cargas y limitar el efecto de eventuales presiones diferenciales, así como garantizar, de forma general, la estabilidad de sistemas constructivos articulados y complejos.
} 
y en los éxitos de una experiencia práctica que, en buena medida, superaría a los planteamientos teóricos. Ello toma aún más sentido valorando la efectista y "teatral", si cabe, arquitectura romana, en la que la complejidad estructural, como pueda ser el caso de esta vulnerabilidad contenida al interior de las piezas, no sólo no afecte a su aspecto exterior sino que, además, trate de ser disimulada cuidadosamente cuando lo compromete.

\section{ALGUNAS CUESTIONES ABIERTAS}

En el estado actual de la investigación, todavía en curso, son muchas aún las cuestiones que deben ser resueltas, sea o no con el auxilio de las nuevas aplicaciones tecnológicas aquí presentadas.

Por un lado, en cuanto al proceso metodológico, es preciso mantener en el ámbito de debate la necesidad de plantear un ajuste entre los recursos tecnológicos utilizados en el estudio y las necesidades planteadas con objeto de evitar el predomino de los medios sobre los fines. En este sentido, la evaluación crítica de los medios es siempre beneficiosa.

Por otro, en cuanto a los fines del estudio, es importante llegar a entender en qué medida pudo ser más rentable recurrir a los complejos recursos técnicos investigados para la época romana, con una elevada inversión en operarios cualificados, instrumental y materiales (e.g. metal transformado) que desechar las piezas. Cabría igualmente preguntarse cuándo se habría tomado conciencia de la debilidad de las mismas, y si habrían sido comercializadas y enviadas a la obra como material intacto de primera calidad. De no ser así, quizá sería de esperar la existencia de circuitos encargados de comercializar productos de menor calidad o subproductos, aunque, en el caso que nos ocupa, un gran edificio público de carácter religioso y connotaciones incluso político-ideológicas, no deja de ser un tanto sorprendente.

La relativa abundancia de estos recursos en diferentes edificios italicenses, hace preguntarse por posibles problemáticas específicas de carácter estructural que pudieran afectarles; en ese sentido y, a pesar de tratarse de un argumento muy controvertido en la historia de la ciudad y cada vez más cuestionado, cabe recordar el eventual efecto nocivo que pudieron ejercer las arcillas (desde el punto de vista geológico Fm margas azules) subyacentes, calificadas tradicionalmente de "expansivas". Al respecto, no deja de ser atractivo tener en cuenta que en diferentes zonas del Imperio ha podido constatarse la adopción de estrategias específicas antisísmicas en arquitectura (Ismaelli 2013: 270), tanto tras sus deterioros efectivos como con carácter preventivo.

En cualquier caso y como conclusión principal, a la espera de nuevos datos, puede afirmarse con rotundidad que se trata de soluciones técnicas asociadas a medidas preventivas de refuerzo y nunca de reparaciones posteriores de elementos fragmentados o deteriorados. Sólo de forma por el momento preliminar cabe proponer su realización asociada, con mayor probabilidad, a la obra de construcción mejor que a la cantera de origen, a partir de un real y efectivo conocimiento por parte de sus responsables de las problemáticas estructurales a las que las piezas estarían sometidas en su destino final.

\section{AGRADECIMIENTOS}

Este trabajo se enmarca en los Proyectos de Investigación del Plan Nacional I+D+i 2012 del Ministerio de Economía y Competitividad: Urbes en transformación. Definición del paisaje urbano de las ciudades romanas del valle del Guadalquivir a través del análisis de las soluciones arquitectónicas: materiales, técnicas y esquemas productivos (HAR2012-36963-C05-04) y Un Modelo Digital de Información para el Conocimiento $y$ Gestión de Bienes Inmuebles del Patrimonio Cultural (HAR2012-34571).

Nuestro agradecimiento a diferentes colegas por sus amables observaciones al respecto de los materiales y recursos estudiados: Lyne Lancaster, Carla M. Amici, Tommaso Ismaelli, Clemens Voigts y Marco Galli, y al Conjunto Arqueológico de Itálica por su disponibilidad para el desarrollo de la investigación.

\section{BIBLIOGRAFÍA}

Amici, C. M. 2011: “L'utilizzazione delle catene metalliche nei sistemi voltati di età romana", en, C. Giardino (ed.), Archeometallurgia: dalla conoscenza alla fruizione, pp. 221-228. Edipuglia, Bari.

Amici, C. M. 2015: "Hidden iron: high tech devices in Roman imperial architecture", Proceedings of the 5th International Congress on Construction History, pp. 63-70. CHSA, Chicago.

Amici, C. M. 2016: Architettura romana. Dal cantiere all'architetto: soluzioni concrete per idee progettuali, Bibliotheca Archaeologica, 53, L'Erma di Bretschneider, Roma.

Baccini Leotardi, P. 1979: Marmi di cava rinvenuti a Ostia e considerazioni sul commercio dei marmi in età romana, Scavi di Ostia, X. Istituto Poligrafico e Zecca dello Stato, Roma. 
Baccini Leotardi, P. 1989: Nuove testimonianze sul commercio dei marmi in età imperiale, Istituto Italiano per la Storia Antica, fasc. 44. Roma.

Caballos, A. (ed.) 2010: Itálica-Santiponce. Municipium y Colonia Aelia Augusta Italica, Ciudades romanas de Hispania. L'Erma di Bretschneider, Roma.

Ismaelli, T. 2013: “Ancient architectural restoration in Asia Minor. Typology, techniques and meanings discussed with reference to examples of largescale public buildings in Hierapolis of Phrygia, a seismic city in Western Turkey", Istanbuler Mitteilungen 63, pp. 267-324.

Jiménez Martín, A. 1989: "Las columnas del teatro de Itálica", Homenaje al Profesor Antonio Blanco Freijeiro, pp. 277-318. Editorial Complutense, Madrid.

Jiménez Martín, A. 1994: "El arquitecto en Roma”, Artistas y artesanos en la Antigüedad clásica. Cuadernos Emeritenses 8, pp. 29-71.

León, P. 1988: Traianeum de Itálica. Monte de Piedad y Caja de Ahorros de Sevilla, Sevilla.

Pensabene, P. 1995: Le vie del marmo. I blocchi di cava di Roma e di Ostia. Il fenomeno del marmo nella Roma antica. Ministero per i beni culturali e ambientali, Soprintendenza archeologica di Ostia, Roma.

Pinto, F., Guerrero, J. M. y Angulo, R. 2011: "Metholology and resources used in the anastylosis proposal for the scaenae frons of the Roman theatre of Italica", Italica. Revista de Arqueología Clásica de Andalucía 1, pp. $77-83$ y pp. $230-232$

Rodríguez, O. 2001: “La reparación de elementos arquitectónicos en época romana: la evidencia en fustes de columna procedentes del teatro romano de Itálica”, Madrider Mitteilungen 42, pp. 138-154, láms. 14-17.

Rodríguez, O. 2015: “«Aquí no se tira nada». Más sobre las dinámicas preventivas en elementos arquitectónicos marmóreos italicenses: refuerzos, reutilización y mercado de ocasión”, en, J. García Sánchez, I. Mañas y F. Salcedo (eds.), Navigare necesse est. Estudios en homenaje a José María Luzón, pp. 365-376. Univ. Complutense de Madrid, Madrid.

Voigts, C. 2012: "Das zweigeschossige Peristyl der Domus Augustana: Untesuchung der Stein-Bauteile und Rekonstruktion der Portiken", en, N. Sojc (ed.), Domus Augustana. Neue Forschungen zum "Versenkten Peristyl" auf dem Palatin, pp. 139-184. Sidestone Press, Leiden.

Voigts, C. e. p.: "High-Tech für den römischen Kaiserpalast: Hohlbohrer und Gewölbeanker", Werkspuren. Diskussionen zur Archäologischen Bauforschung 12 (2016), Berlín.

Wurch-Kozelj, M. 1988: "Methods of Transporting Blocks in Antiquity", en, N. Herz, y M. Waelkens (eds.), Classical Marble: Geochemistry, Technology, Trade, pp. 55-64. Springer, Dordrecht. 